\title{
Therapie der Rheumatoiden Arthritis: Was bringen Ultraschall und Biomarker?
}

Patienten mit rheumatoider Arthritis bleiben offensichtlich nicht häufiger in Remission, wenn Ultraschallbefunde in Therapieentscheidungen einbezogen werden. Sind Biomarker bei der Definition „Remission“ ebenfalls verzichtbar?

\begin{abstract}
Bei der Behandlung von Patienten mit rheumatoider Arthritis (RA) werde heute ausdrücklich empfohlen, im Rahmen einer Treat-to-Target (T2T)Strategie konkrete Therapieziele festzulegen. Hierauf verwies Dr. Alexander Sepriano, Leiden, Niederlande, demzufolge die klinische Remission ein mögliches Therapieziel sei. Im Ultraschall sind dann aber oft noch Zeichen einer subklinischen Synovitis erkennbar, die mit einer radiologischen Progression einhergeht und das Risiko des Wiederaufflackerns der klinischen Symptomatik („Flare“) erhöht [1, 2].
\end{abstract}

》) Zwei randomisierte Studien konnten keinen Nutzen der Ultraschallgestützten Therapie nachweisen

Vor diesem Hintergrund haben Sepriano und Kollegen in der prospektiven multizentrische BIODAM-Studie die Frage geprüft: Bleiben mehr Patienten klinisch in Remission, wenn im Rahmen der T2T-Strategie - zusätzlich zur klinischen Untersuchung - sieben stark betroffene Gelenke an Händen und Füßen mittels Ultraschall untersucht werden und bei Zeichen einer Synovitis eine Modifikation der Therapie erfolgt?

\section{Schon die dritte Studie mit negati- vem Ausgang}

Die 130 eingeschlossenen Patienten wurden bei $41 \%$ der Visiten sowohl klinisch als auch mit Ultraschall untersucht. Drei Monate später waren sie aber nicht häufiger in klinischer Remission als bei alleiniger klinischer Untersuchung. Im Gegenteil: Gemäß den Kriterien des American College of Rheumatology (ACR) und der European League Against Rheumatism (EULAR) war die Wahrscheinlichkeit sogar deutlich geringer. Wie Sepriano einräumte, war die Studie nicht randomisiert. Zwei randomisierte kontrollierte Studien konnten aber ebenfalls keinen Nutzen der Ultraschall-gestützten Therapie nachweisen $[3,4]$.

》) Das Ausmaß der Gelenkschwellung ist ein stärkerer Prädiktor der Progression als die Höhe des CRP

Dies zeige aus Sicht von Prof. Espen A. Haavardsholm, Oslo, Norwegen, wie wichtig es sei, auch nicht-medikamentöse Interventionen unter kontrollierten Bedingungen zu prüfen. Subklinische Entzündung bei RA lasse sich behandeln. Mittels Bildgebung darstellbare Remission sei seines Erachtens aber kein besseres Therapieziel als die klinische Remission.

\section{Biomarker bestimmen?}

\section{Pro und Contra}

Die Bedeutung von Entzündungsmarkern als Entscheidungshilfe in der RATherapie nahm PD Dr. Daniel Aletaha, Wien, kritisch unter die Lupe. Zusammen mit Kollegen führte er Untersuchungen durch, denen zufolge das Ausmaß der Gelenkschwellung bei Patienten mit nahezu vollständiger Remission ein stärkerer Prädiktor der Progression ist als die Höhe des C-reaktiven Proteins (CRP) [5]. Würden bei einem Patienten in klinischer Remission die Therapiemaßnahmen intensiviert, um auch die Biomarker in den Normbereich zu bringen, sei dies mit höheren Kosten verbunden. Das Outcome für die Patienten verbessere der Mehraufwand nicht.

Aus Sicht von Prof. Dr. Ronald van Vollenhoven, Amsterdam, Niederlande, sind Biomarker aber keinesfalls verzichtbar: „Die rheumatoide Arthritis ist ein biologischer Prozess - und deshalb ist es sinnvoll, Biomarker in die Definition für Remission mit einzubeziehen", betonte der Rheumatologe. In der täglichen Praxis gehe es immer wieder um die Frage, ob ein Medikament in der Dosis reduziert oder ausschleichend abgesetzt werden könne. Hier seien Biomarker von unschätzbarem Wert, um die richtigen Entscheidungen treffen zu können.

\section{Literatur}

1. Brown AK et al (2008) An explanation for the apparent dissociation between clinical remission and continued structural deterioration in rheumatoid arthritis. Arthritis Rheum 58:2958-2967

2. Saleem B et al (2012) Can flare be predicted in DMARD treated RA patients in remission, and is it important? A cohort study. Ann Rheum Dis 71:1316-1321

3. Dale J et al (2016) Targeting ultrasound remission in early rheumatoid arthritis: the results of the TaSER study, a randomised clinical trial. Ann Rheum Dis 75:1043-1050

4. Haavardsholm EA et al (2016) Ultrasound in management of rheumatoid arthritis: ARCTIC randomised controlled strategy trial. BMJ 354:i4205

5. Aletaha D, Alasti F, Smolen JS (2011) Rheumatoid arthritis near remission: clinical rather than laboratory inflammation is associated with radiographic progression. Ann Rheum Dis 70:1975-1980

rheuma plus $2017 \cdot 16: 82$

DOI 10.1007/s12688-017-0129-z

(c) Springer-Verlag GmbH Austria 2017 